\title{
An equivalent dipole analysis of PZT ceramics and lead-free piezoelectric single crystals
}

\author{
Andrew J. Bell \\ Institute for Materials Research, School of Chemical and Process Engineering \\ University of Leeds, Leeds, LS2 9JT, UK
}

a.j.bell@leeds.ac.uk

\begin{abstract}
Received 21 December 2015; Revised 11 February 2016; Accepted 15 February 2016; Published 13 April 2016
The recently proposed Equivalent Dipole Model for describing the electromechanical properties of ionic solids in terms of 3 ions and 2 bonds has been applied to PZT ceramics and lead-free single crystal piezoelectric materials, providing analysis in terms of an effective ionic charge and the asymmetry of the interatomic force constants. For PZT it is shown that, as a function of composition across the morphotropic phase boundary, the dominant bond compliance peaks at $52 \% \mathrm{ZrO}_{2}$. The stiffer of the two bonds shows little composition dependence with no anomaly at the phase boundary. The effective charge has a maximum value at $50 \% \mathrm{ZrO}_{2}$, decreasing across the phase boundary region, but becoming constant in the rhombohedral phase. The single crystals confirm that both the asymmetry in the force constants and the magnitude of effective charge are equally important in determining the values of the piezoelectric charge coefficient and the electromechanical coupling coefficient. Both are apparently temperature dependent, increasing markedly on approaching the Curie temperature.
\end{abstract}

Keywords: Piezoelectricity; ferroelectricity; modeling.

\section{Introduction}

Piezoelectric materials are unsurpassed in electromechanical transduction applications in fields such as ultrasound medical imaging, sonar, pressure sensing and actuation. ${ }^{1}$ Their importance is emphasized by the significant effort in research into new piezoelectric materials, aimed at either improving performance, for example in lead-based single crystals, ${ }^{2}$ or identifying systems that will meet potential environmental legislation. ${ }^{3}$ Much of the research focuses on either identifying systems with a morphotropic phase boundary, in order to replicate the performance of the market leading material $\mathrm{Pb}(\mathrm{Zr}, \mathrm{Ti}) \mathrm{O}_{3},{ }^{4}$ or in optimizing domain wall contributions to piezoelectric activity. ${ }^{5}$ Experimental efforts are supported by excellent models, for example thermodynamic theory in the case of morphotropic phase boundaries ${ }^{6}$ and domain wall dynamics in the case of extrinsic contributions. ${ }^{7}$ However, there is little guidance from current theories in identifying baseline systems that may have high intrinsic piezoelectric activity. The thermodynamic ${ }^{8}$ and lattice dynamics ${ }^{9}$ models of ferroelectricity are not sufficiently predictive to provide guidance to experimentalists. Whilst, Density Functional Theory is developing rapidly, to the point where the piezoelectric properties may be predicted for specific compositions, ${ }^{10}$ such calculations currently carry a high computational overhead and they are not yet able to rapidly screen a large number of compounds with sufficient accuracy to guide experimental surveys.

In order to provide a more intuitive approach for analyzing the piezoelectric behavior of materials and specifically taking into account the relationships between the primary material coefficients, a simple classical mechanics model has been proposed. ${ }^{11}$ The model is based on the simplest structural motif necessary to provide piezoelectric behavior: a 3 atom dipole defined by an effective ionic charge and asymmetry in the strength of the interatomic bonds. Applying this Equivalent Dipole Model to the property data for any piezoelectric material, both ferroelectric and non-ferroelectric, provides an evaluation of the effective charge and bond strengths, which can be used as simple parameters to describe and compare the origin of the piezoelectric effect in that material.

The model employs the 3 atom motif as a surrogate for the much more complex structures that usually give rise to piezoelectricity, but due to its simplicity it may provide effective guidance to experimental approaches for the discovery of new piezoelectric materials. The model is somewhat analogous to the equivalent circuit approach to model the electrical impedance of materials, or the spring, lever and damper approach to model complex mechanical systems. In neither of these cases do we believe that the systems comprise the actual components of the model, but we do value the information the model provides to help us understand the more complex actuality of the system.

Here, by applying the model to two sets of materials, $\mathrm{Pb}(\mathrm{Zr}, \mathrm{Ti}) \mathrm{O}_{3}$ ceramic compositions across the morphotropic phase boundary and lead-free single crystals, we examine to what extent the 3 atom model may be able to help identify new or improved piezoelectric materials.

This is an Open Access article published by World Scientific Publishing Company. It is distributed under the terms of the Creative Commons Attribution 4.0 (CC-BY) License. Further distribution of this work is permitted, provided the original work is properly cited. 


\section{Theory}

The model is based on a static electromechanical analysis of the 3 atom dipole as shown in Fig. 1, which comprises two ions with charge $-q$ separated by distance $a$. A third ion of charge $+2 q$ is displaced from the mid-point by a distance $\delta$ with bonds between the three ions characterized by inverse force constants $\gamma_{1}$ and $\gamma_{2}$. It is assumed the dipole occupies a characteristic volume, $v$. For clarity, the inverse force constants are generally defined by $\Delta a=\gamma F$, where $\Delta a$ is the increase in length of the bond when subjected to a force $F$.

A static analysis of the changes in ion positions when the dipole is subjected to a force or electric field parallel to its length yields the following relationships:

$$
\begin{gathered}
P_{s}=2 q \delta / v, \\
s^{E}=\frac{v}{a^{2}}\left(\gamma_{1}+\gamma_{2}\right), \\
\varepsilon^{X} \approx \varepsilon_{0} \chi^{X}=\frac{q^{2}}{v}\left(\gamma_{1}+\gamma_{2}\right)
\end{gathered}
$$

and

$$
d=\frac{q}{a}\left(\gamma_{1}-\gamma_{2}\right)
$$

where $P_{s}$ is the dipole moment per unit volume, equivalent to the spontaneous polarization, $s^{E}$ is the elastic compliance at constant electric field, $\varepsilon^{X}$ is the dielectric permittivity at constant stress and $d$ is the piezoelectric charge coefficient. The dielectric susceptibility $\chi^{X}$ is considered equal to the relative permittivity $\varepsilon^{X} / \varepsilon_{0}$ (where $\varepsilon_{0}$ is the permittivity of free space) for cases where $\varepsilon^{X}>10 \varepsilon_{0}$; this approximation is applied consistently here, unless stated otherwise. It should be noted that none of the three primary coefficients depend on the displacement $\delta$, confirming that ferroelectricity is not a prerequisite for piezoelectricity.

Combining the three coefficients according to the relationship for the electromechanical coupling coefficient $k$ :

$$
k=\sqrt{\frac{\text { converted energy }}{\text { input energy }}}=\frac{d}{\sqrt{s^{E} \varepsilon^{X}}}=\frac{\gamma_{1}-\gamma_{2}}{\gamma_{1}+\gamma_{2}} .
$$

The electromechanical coupling coefficient is often referred to as "the best single measurement of the strength of the piezoelectric effect", ${ }^{4}$ hence it is most instructive to see that the model suggests that it is dependent only on the

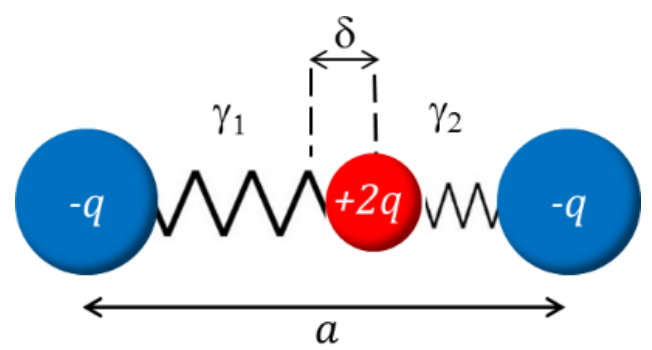

Fig. 1. The 3 atom dipole used as the basis of the model, with ionic charge $q$, length $a$, bond compliances $\gamma_{1}$ and $\gamma_{2}$ and a dipole offset $\delta$. asymmetry in the bond strengths within the 3 atom motif. If extended to real crystals, this would suggest that bond asymmetry is more important than the valence states of the constituent ions.

In Ref. 11, it was shown that the intuitive interpretation of the relationship between $k$ and the three material coefficients, which implies that $k$ is inversely proportional to compliance and permittivity, is misleading. Due to the interdependence of the three coefficients embodied in Eqs. (2)-(4), $k$ actually increases with increasing $s^{E}$ and $\varepsilon^{X}$.

From Eqs. (2)-(4), if the three longitudinal coefficients $\left(s_{33}^{E}, \varepsilon_{33}^{X}\right.$ and $\left.d_{33}\right)$ are known for a given material, along with the lattice parameters, values for $q, \gamma_{1}$ and $\gamma_{2}$ can be evaluated as follows:

$$
\begin{gathered}
q=\frac{v}{a} \sqrt{\frac{\varepsilon_{33}^{X}}{s_{33}^{E}}} \\
\gamma_{1}=\frac{1}{2}\left(\frac{a^{2}}{v} s_{33}^{E}+\frac{a}{q} d_{33}\right)
\end{gathered}
$$

and

$$
\gamma_{2}=\frac{1}{2}\left(\frac{a^{2}}{v} s_{33}^{E}-\frac{a}{q} d_{33}\right) .
$$

The use of these relationships was validated in Ref. 11 using a wide range of piezoelectric materials from low- $k$, nonferroelectric materials, through PZT ceramics to high- $k$ single crystals. It was shown that whilst the effective charge $q$ was important in determining the size of the piezoelectric effect, the best piezoelectric materials were characterized by very large asymmetry in the bond strengths, with the most compliant bond dominating the electromechanical behavior.

\section{Results}

\section{1. $\mathrm{Pb}(\mathrm{Zr}, \mathrm{Ti}) \mathrm{O}_{3}$ ceramics}

The archetypal piezoelectric ceramic, $\mathrm{Pb}\left(\mathrm{Zr}_{x} \mathrm{Ti}_{1-x}\right) \mathrm{O}_{3}$, or PZT, has been studied extensively in order to understand the mechanisms of piezoelectricity which lead to its class leading performance. Both the coupling coefficient and charge coefficient peak at approximately $x=0.52$, almost coincident with a phase boundary between the perovskite rhombohedral and tetragonal symmetries.

Figures 2(a) and 2(b) show the charge coefficient, coupling coefficient, permittivity and compliance of PZT as a function of $\mathrm{Zr}$ concentration as determined by Berlincourt et al. ${ }^{12}$ The $d_{33}$ coefficient, coupling coefficient and elastic compliance all peak at $x=0.52$. However, the permittivity peaks at approximately $x=0.50$.

Equations (6)-(8) were used to calculate the model parameters from the results shown in Figs. 2(a) and 2(b). These are shown in Figs. 2(c) and 2(d). Whilst $x=0.52$ is often regarded as the MPB composition, due to the peak in $d_{33}$ and $k_{33}$, the tetragonal to rhombohedral symmetry change 


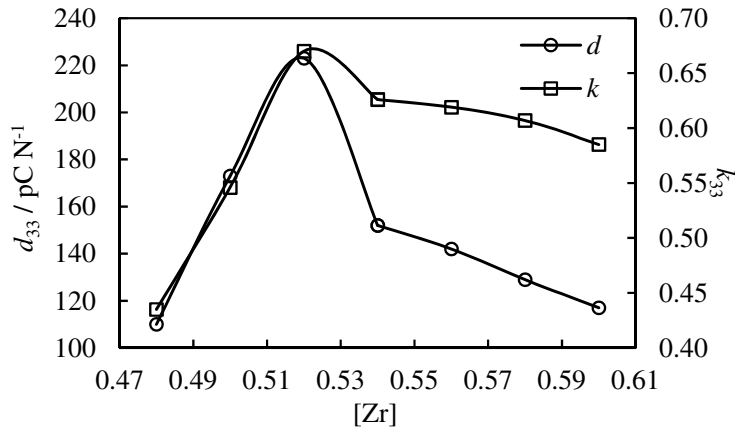

(a)

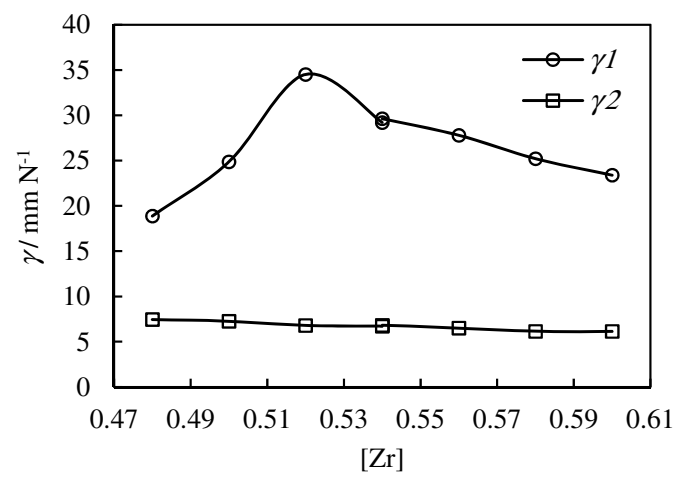

(c)

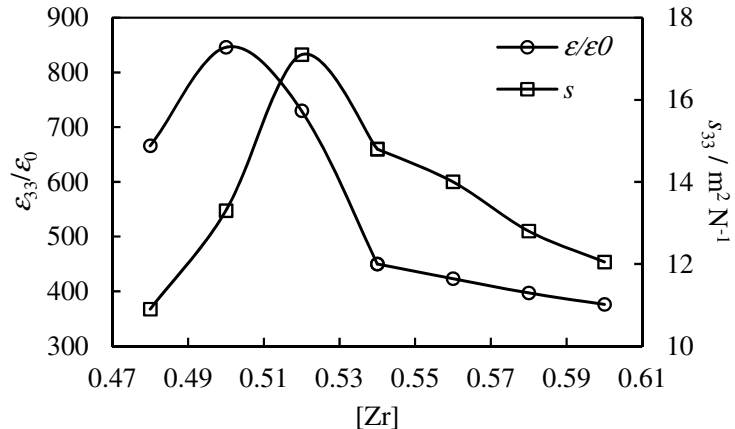

(b)

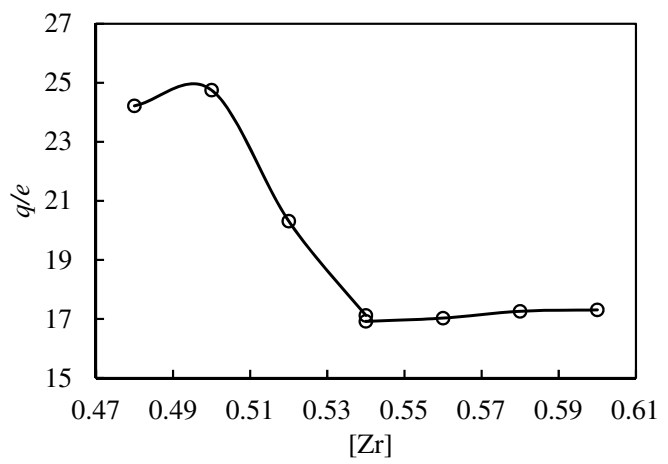

(d)

Fig. 2. (a) Measured values for $d_{33}$ and $k_{33}$, and (b) $\varepsilon_{33} / \varepsilon_{0}$ and $s_{33}$ for $\mathrm{Pb}(\mathrm{Zr}, \mathrm{Ti}) \mathrm{O}_{3}$ as a function of zirconium concentration after Ref. 12; (c) bond compliances $\gamma_{1}$ and $\gamma_{2}$ and (d) effective charge $q$ calculated from the same data.

reported in Ref. 12 is at $x=0.54$, where the two phases coexist. For this reason, the model parameters were evaluated for both tetragonal and rhombohedral symmetry for the data at $x=0.54$; both results are shown in Figs. 2(c) and 2(d). The difference between these results is minimal with the points overlapping at the scale shown. The effective charge, $q$, peaks at $x=0.5$ with a value of $25 e$, where $e$ is the electron charge, and is approximately constant at $q=17 e$ in the rhombohedral phase for $x>0.53$ Whilst the value of $\gamma_{2}$ remains approximately constant at $\sim 8 \mathrm{~mm} \mathrm{~N}^{-1}$, the value of $\gamma_{1}$, the softer of the two bonds, peaks at $x=0.52$, with a value of $35 \mathrm{~mm} \mathrm{~N}^{-1}$. Hence, whilst the effective charge peaks in the tetragonal phase, it is the bond asymmetry which determines that the optimum piezoelectric properties are found at $x=0.52$.

Whilst it is probably not incorrect to assume that there is intrinsic lattice softening around the MPB, the role of domain wall motion also cannot be ignored. As previously discussed, ${ }^{11}$ the equivalent dipole analysis cannot distinguish between bond softening and domain wall mobility, hence it must be accepted that increased domain wall mobility at the MPB also contributes to the peak in $\gamma_{1}$.

\subsection{Lead-free single crystals}

To attempt to identify trends in the model parameters, literature data has been collected for a number of lead- free single crystals as shown in Fig. 3. The materials are $\mathrm{LiNbO}_{3},{ }^{13} \quad \mathrm{LiTaO}_{3},{ }^{13} \quad \mathrm{KNbO}_{3},{ }^{14} \mathrm{BaTiO}_{3} \quad$ (BT), ${ }^{4}$ $(\mathrm{K}, \mathrm{Na})\left(\mathrm{Nb}, \mathrm{Ta}^{\mathrm{O}} \mathrm{O}_{3}(\mathrm{KNNT}),{ }^{15} \mathrm{Li} \& \mathrm{Ta}\right.$-doped $(\mathrm{K}, \mathrm{Na}) \mathrm{NbO}_{3}$ $(\mathrm{KNN}-\mathrm{LT})^{16}$ and $\left(\mathrm{Na}_{1 / 2} \mathrm{Bi}_{1 / 2}\right) \mathrm{TiO}_{3}-\mathrm{BaTiO}_{3}(\mathrm{NBT}-\mathrm{BT}){ }^{17}$ and have been ordered in increasing value of permittivity along the $x$-axis of Fig. 3.

Except in two cases, the measured $s_{33}, d_{33}$ and $k_{33}$ values follow the trend of $\varepsilon_{33}$. The exceptions are NBT-BT, in which $d_{33}$ and $k_{33}$ are lower than the trends that $\varepsilon_{33}$ and $s_{33}$ suggest, whilst in $\mathrm{BaTiO}_{3}$, the $k_{33}$ is lower and the $s_{33}$ is higher than suggested by the trend in $\varepsilon_{33}$. The corresponding model parameters in Fig. 3(c) all show an increasing trend along the $x$-axis; that is, $q, \gamma_{1}$ and $\gamma_{1}-\gamma_{2}$ increase. It can be seen that the two exceptions of $\mathrm{BaTiO}_{3}$ and NBT-BT are caused by $q$ being above the trend in $\mathrm{BaTiO}_{3}$ and below the trend in NBT-BT.

These data appear to confirm the findings of Ref. 11, that, in general, all three of the primary electromechanical coefficients $\left(s_{33}^{E}, \varepsilon_{33}^{X}\right.$ and $\left.d_{33}\right)$ increase with increasing $k_{33}$ and that to achieve the best piezoelectric performance both a large $q$ and a large $\gamma_{1}\left(\gg \gamma_{2}\right)$ are required. However the importance of Curie temperature $\left(T_{\mathrm{C}}\right)$ in making such comparisons cannot be ignored. With the exception of $\mathrm{BaTiO}_{3}$, the trend of increasing permittivity in Fig. 3(a) strongly follows the reciprocal of Curie temperature. Whilst the data support the assumption that both $q$ and $\gamma_{1}$ increase on approaching $T_{\mathrm{C}}$, it 


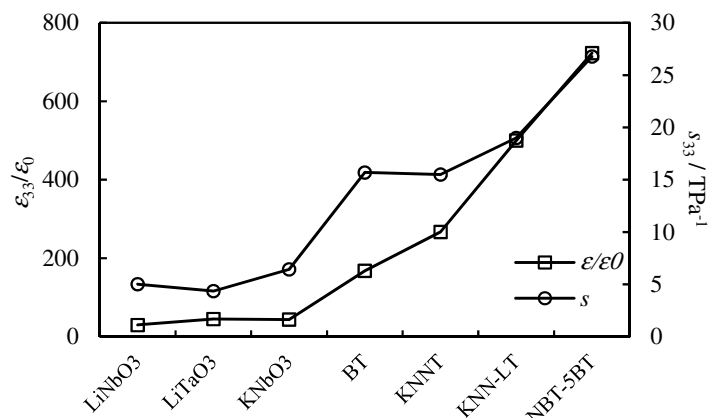

(a)

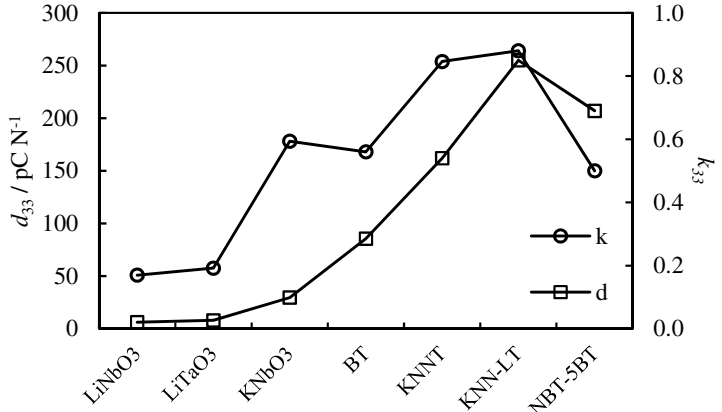

(b)

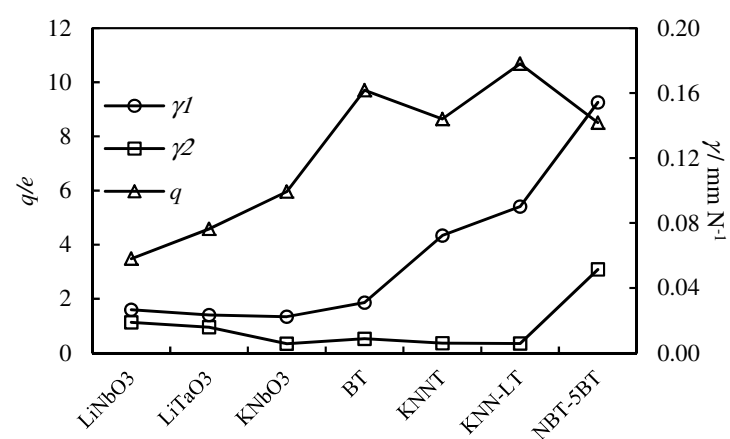

(c)

Fig. 3. (a) Measured values for $\varepsilon_{33} / \varepsilon_{0}$ and $S_{33}$, (b) $d_{33}$ and $k_{33}$ for various lead-free single crystals (see text) and (c) $q$, $\gamma_{1}$ and $\gamma_{2}$, calculated from the data.

begs the question of which parameter has the more dominant temperature dependence.

The Landau Devonshire theory of ferroelectrics provides a means of predicting the temperature dependence of both the permittivity and the charge coefficient, under the assumption that the electrostriction coefficient is temperature independent. However, it has often been assumed, when applying the theory to piezoelectricity, the elastic compliance is independent of temperature. This is an obvious short-coming in the model as an early measurement of the compliance of $\mathrm{BaTiO}_{3}$ show a significant divergence at the Curie temperature. ${ }^{18}$ A further analysis of the temperature dependence of all three primary coefficients for a model system would allow a more detailed understanding of the temperature dependence of the model parameters.

\section{Conclusions}

The Equivalent Dipole model has been applied to PZT ceramics and lead-free single crystal piezoelectric materials. For PZT it is shown that the dominant bond compliance and effective charge peak close to the MPB, whilst the stiffer of the two bonds shows little composition dependence. The single crystals confirm that both the asymmetry in the force constants and the magnitude of effective charge are equally important in determining the piezoelectric properties. Both are apparently temperature dependent, increasing markedly on approaching $T_{C}$.

Whilst the 3 atom model shows some promise in aiding materials scientists to interpret results and seek new piezoelectric materials, its utility is currently limited by a number of issues: (i) difficulties in validation by independent measurement of the model parameters, (ii) a small existing data set from which to extrapolate to new compositions and (iii) harmonization with other models which would allow an understanding of how the model parameters, and hence material properties, vary as a function of external variables.

In terms of validation, the major implication of the model, that the magnitude of piezoelectric activity is function of asymmetry in sub-unit cell bonding, could be tested by careful in situ diffraction experiments. That is, the change of atomic positions as a function of applied uniaxial stress could be determined by X-ray or neutron diffraction for representative piezoelectric materials (e.g., $\mathrm{BaTiO}_{3}$ versus PMN-PT), allowing an analysis of the relevant bond compliances. These experiments are not simple and may take some time to accomplish. However, the same "experiments" are achievable in silicio and DFT investigations are currently being undertaken to this end. To a limited extent, a form of experimental validation was demonstrated in Ref. 11 in which it was shown that the value of $\delta$ calculated from the model scales with the measured atomic displacements responsible for spontaneous polarization in $\mathrm{BaTiO}_{3}, \mathrm{PbTiO}_{3}$ and $\mathrm{KNbO}_{3}$. 
If sufficient self-consistent data were available for a wide range of compounds, it is tempting to postulate that the equivalent dipole model could be part of a methodology for new materials discovery, through the identification of element combinations and structural motifs, beyond the ubiquitous perovskites, that give rise to large bond strength asymmetry. For example, although the ionic radius of an ion is ill-defined in a single experiment, tables of ionic radii can be compiled by inference from a large crystallographic database. Likewise, given sufficient piezoelectric data, inferential assignment of the model parameters to compositional and structural motifs would be possible and would play a role in new materials discovery. Nevertheless, a less resource intensive computational analysis of the detailed mechanisms of piezoelectricity in representative materials, guided by the lessons of the equivalent dipole model, could contribute significantly to future digital search strategies.

Finally, as alluded to above, the equivalent dipole model can be both expanded to provide additional analysis and be harmonized with other models which address piezoelectricity. For example, Ref. 11 has already shown how nonlinear bond strengths are relevant to a broader range of electromechanical properties including electrostriction. One can also postulate that the extension to include $\gamma$ as a complex quantity may allow some additional insights into both the origins of electromechanical losses and allow prediction of the frequency dependence of the intrinsic properties. A nonlinear and frequency-dependent model would also allow separation of intrinsic and extrinsic piezoelectric mechanisms. A threedimensional model may also be postulated, most probably a 7 atom dipole, from which additional tensor elements could be derived from the simple one-dimensional primary coefficient measurements.

\section{References}

${ }^{1}$ A. Safari and E. K. Akdogan (eds.), Piezoelectric and Acoustic Materials for Transducer Applications (Springer, USA, 2009).

${ }^{2} \mathrm{~S}$. Zhang and F. Li, High performance ferroelectric relaxor$\mathrm{PbTiO}_{3}$ single crystals: Status and perspective, Appl. Phys. Lett. 111, 031301 (2012).

${ }^{3}$ J. Roedel, W. Jo, K. T. P. Seifert, E. M. Anton, T. Granzow and D. Damjanovic, J. Am. Ceram. Soc. 92, 1153 (2009).
${ }^{4}$ B. Jaffe, W. R. Cook and H. Jaffe, Piezoelectric Ceramics (Academic Press, London, 1971).

${ }^{5}$ S. Wada, S. Suzuki, T. Noma, T. Suzuki, M. Osada, M. Kakihana, S. E. Park, L. E. Cross and T. R. Shrout, Enhanced piezoelectric property of barium titanate single crystals with engineered domain configurations, J. Appl. Phys. 38, 5505 (1999).

${ }^{6}$ A. A. Heitmann and G. A. Rossetti, Thermodynamics of ferroelectric solid solutions with morphotropic phase boundaries, J. Am. Ceram. Soc. 97, 1661 (2014).

${ }^{7}$ D. Damjanovic and M. Demartin, Contribution of the irreversible displacement of domain walls to the piezoelectric effect in barium titanate and lead zirconate titanate ceramics, J. Phys. Condens. Matter. 9, 4943 (1997).

${ }^{8}$ A. F. Devonshire, Theory of ferroelectrics, Adv. Phys. 3, 85 (1954).

${ }^{9}$ W. Cochran, Crystal stability and the theory of ferroelectricity, Adv. Phys. 9, 387 (1960).

${ }^{10}$ P. Baettig, C. F. Schelle, R. LeSar, U. V. Waghmare and N. A. Spaldin, Theoretical prediction of new high-performance lead-free piezoelectrics, Chem. Mater. 17, 1376 (2005).

${ }^{11}$ A. J. Bell, A classical mechanics model for the interpretation of piezoelectric property data, J. Appl. Phys. 118, 224103 (2015).

${ }^{12}$ D. A. Berlincourt, C. Cmolik and H. Jaffe, Piezoelectric properties of polycrystalline lead titanate zirconate compositions, Proc. IRE 48, 220 (1960).

${ }^{13} \mathrm{~W}$. Yue and J. Yi-jian, Crystal orientation dependence of piezoelectric properties in $\mathrm{LiNbO}_{3}$ and $\mathrm{LiTaO}_{3}$, Opt. Mater. 23, 403 (2003).

${ }^{14}$ S. Wada, K. Muraoka, H. Kakeloto, T. Tsurumi and H. Kumagai, Enhanced piezoelectric properties of potassium niobate single crystals by domain engineering, J. Appl. Phys. 43, 6692 (2004).

${ }^{15}$ L. Zhang, X. Huo, R. Wang, J. Wang, W. Jiang and W. Cao, Large size lead-free $(\mathrm{Na}, \mathrm{K})(\mathrm{Nb}, \mathrm{Ta}) \mathrm{O}_{3}$ piezoelectric single crystal: Gowth and full tensor properties, Cryst. Eng. Comm. 15, 7718 (2013).

${ }^{16}$ X. Huo, L. Zheng, R. Zhang, R. Wang, J. Wang, S. sang, Y. Wang, B. Yang and W. Cao, A high quality lead-free (Li,Ta) modified $(\mathrm{K}, \mathrm{Na}) \mathrm{NbO}_{3}$ single crystal and its complete set of elastic, dielectric and piezoelectric coefficients with macroscopic $4 \mathrm{~mm}$ symmetry, Cryst. Eng. Comm. 16, 9828 (2014).

${ }^{17}$ K-S. Moon, D. Rout, H-Y. Lee and S-J. L. Kang, Solid state growth of $\mathrm{Na}_{1 / 2} \mathrm{Bi}_{1 / 2} \mathrm{TiO}_{3}-\mathrm{BaTiO}_{3}$ single crystals and their enhanced piezoelectric properties, J. Cryst. Crowth 317, 28 (2011).

${ }^{18}$ E. J. Huibregtse, W. H. Bessey and M. E. Drougard, Electromechanical behaviour of single crystals of barium titanate from $25^{\circ} \mathrm{C}$ to $160^{\circ} \mathrm{C}$, J. Appl. Phys. 30, 899 (1959). 